\title{
Analyses of Crawford's uvby $\beta$ calibrations using the pulsational variations of FG Vir
}

\author{
P. Haas $^{1}$ \\ ${ }^{1}$ Institut für Astronomie, Türkenschanzstrasse 17, A-1180 Vienna, Austria
}

\begin{abstract}
Crawford's uvby $\beta$ calibration method is examined for A-type stars by comparing it with the pulsational variations of the observable $m_{1}, c_{1}$ and $M_{V}$ for the $\delta$ Scuti star FG Vir. The fit between the calibration values of $m_{1}$ and $M_{V}$ and the respective measurements for FG Vir are tested as a function of temperature based on 3068 4-colour values taken at the Observatorio de Sierra Nevada in Spain during the years 2002 and 2003 . Testing is performed by means of linear regression. The fit between the measured index $m_{1}$ of FG Vir and the $m_{1}$ index of the Hyades is nearly perfect. A fit between the calibration value $M_{V}$ and the measured values of FG Vir cannot be obtained with Crawford's calibration procedure in a straightforward manner. In order to achieve an optimal fit for $M_{V}$ two modifications of the calibration procedure are investigated and discussed. (i) the position of the ZAMS given by Crawford is replaced by the position of the ZAMS given by Mermilliod; (ii) the influence of the mass difference on $c_{1}$ is taken into account.
\end{abstract}

Accepted: November 5, 2010

Individual Objects: FG Vir

\section{Introduction}

About five decades ago, Bengt Strömgren (1963, 1966) developed the uvby intermediate-band photometric system, which became a widely used tool for determining fundamental parameters of stars. First calibrations of the system were carried out by Strömgren and Crawford (1966, 1975, 1978, 1979) and by Perry, Olsen and Crawford (1987). So far, all studies were based on a large number of stars or sets of stars in order to improve the calibration of the 
parameters. Several investigations have been reported in the past on how the Strömgren parameters $m_{1}$ und $c_{1}$ behave during the pulsational variations of a single variable star. Rodríguez et al. (1991) investigated the behaviour of $m_{1}$ for several $\delta$ Scuti stars, Rodríguez et al. $(1992,1998,2003)$ the behaviour of $m_{1}$ and $c_{1}$ for more $\delta$ Scuti stars, Stankov et al. (2002) the behaviour of $m_{1}$ and $c_{1}$ for the star SX Phe.

For the current investigation the $\delta$ Scuti star FG Vir has been used in order to test Crawford's uvby $\beta$ calibrations. Various reasons make this star a suitable candidate, for instance:

a) FG Vir is a pulsating star and during its pulsation the star is varying in $T_{\text {eff }}$, $\log g$, etc., and the star's indices, at each moment, must resemble those of one standard star with the same parameters ( $T_{\text {eff }}, \log g, M_{V}$ and metallicity index $\left.m_{1}\right)$,

b) the data set used is very precise,

c) FG Vir is within the range of validity of Crawford's calibrations.

FG Vir is classified as an A5-type star on the Main Sequence (MS) with $\left\langle\delta c_{1}\right\rangle=0.09 \mathrm{mag}$ as can be seen in Fig. 4; hence $\mathrm{D}(\log g)$ is about 0.4 from ZAMS, and log $g$ of FG Vir is 3.95. Last but not least, the star has nearly solar abundances: $\left\langle\delta m_{1}\right\rangle=0.018$ as can be derived from Fig. 1; hence $[\mathrm{Me} / \mathrm{H}]=-0.11$ follows using Smalley's (1993) calibrations. According to this, one can assume FG Vir as an excellent candidate to test the ZAMS calibrations of Crawford (1979) or similar ones by Philip \& Egret (1980).

Changes of luminosity according to the pulsational phase as well as of log $g$ have to be taken into account for the determination of $\delta c_{1}$ relative to the ZAMS as will be outlined in section 7.. For High Amplitude $\delta$ Scuti (HADS) and SX Phe pulsators see for example the loops of $T_{\text {eff }}$ and $\log g$ in the $\left(c_{1}, b-y\right)$ diagrams during the pulsation cycle in Fig. 4 of Rodríguez et al. (1993), and Fig. 6 of Rodríguez et al. (2003) for V2109 Cyg. For the star SX Phe in Rolland et al. (1991) Fig. 8 shows an analogous behaviour.

Another question which has to be answered is that of the influence of metallicity $[\mathrm{Me} / \mathrm{H}]$. Let us assume a pulsating star similar to FG Vir as far as its location in the HR diagram is concerned but with a quite different metallicity, e.g. $[\mathrm{Me} / \mathrm{H}]=-3.0$. Can we use this star to test Crawford's calibrations for the ZAMS? The answer is negative, because for this star the predictions for $m_{1}$ variations during the pulsation cycle, which imply the dependence on temperature, is very different from the predictions for the ZAMS. The prediction is valid for solar abundances. The predictions by the $\left(m_{1}, \beta\right)$ grids change with $T_{\text {eff }}, \log g$ and most important, with $[\mathrm{Me} / \mathrm{H}]$ as Rodríguez et al. (1991) have shown in Fig. 3, i.e. $m_{1}$ of the star has to follow the predictions given by the corresponding $\left(m_{1}, \beta\right)$ grids. And these predictions are quite different for a ZAMS star with solar abundances. Hence, the $\delta m_{1}$ index must vary during the 
pulsation cycle in agreement with the corresponding predictions. This does not mean that the metallicity of the pulsating star is varying. This means for this star that its $m_{1}$-index behaviour during the pulsation cycle is different from that predicted for a ZAMS star with solar abundances.

Even if we consider a pulsating High Amplitude star on the Main Sequence with solar abundances, we can find variations of $\delta m_{1}$ during the pulsation cycle. During the pulsation the surface gravity of the star is varying as well as its luminosity and the $m_{1}$-index behaviour depends on surface gravity too as shown by Rodríguez et al. (1991) (see, e.g., Fig. 2 and Fig. 4 of his paper). For high amplitude pulsations the dependence $m_{1}=m_{1}(\log g)$ must be taken into account to test the ZAMS calibrations.

To summarize: the metallicity index $m_{1}$ varies with effective temperature and to a less degree with surface gravity.

In the current investigation Strömgren photometric data for the $\delta$ Scuti star FG Vir were collected during 42 nights in the years 2002 and 2003 at the Observatorio de Sierra Nevada. For more details see section 2.

Out of these 42 nights 37 nights have been selected and were combined to an entity of 3068 records, with each record containing the measurements of $u, v, b$ and $y$. These data can be regarded as data from different stars, since FG Vir is changing its surface temperature $T_{\text {eff, }}$, its radius, its surface gravity $g$ etc. during pulsation. On the contrary, the mass of FG Vir and the abundance of all elements remain constant.

Based on these measurements the present paper is testing the calibrations of the metallicity index $m_{1}$, the absolute magnitude $M_{V}$ and the Balmer discontinuity index $c_{1}$ according to the tables and procedures given by Crawford (1979) for A-type stars. The observed values of the measured colours should fit the colours and $M_{V}$ based on Crawford's calibrations. Linear relations between the physical properties of FG Vir are assumed because of its low amplitude pulsating behaviour.

The present paper demonstrates that a fit between the trends of the measured values of $y$ with those of $M_{V}$, determined according to Crawford's calibration procedure, can only be achieved with essential modifications to the calibration procedure. Possible modifications of the calibration procedure are discussed.

\section{Observations}

The investigations in the present paper are based on measurements obtained with the $0.90 \mathrm{~m}$ telescope located at $2900 \mathrm{~m}$ above sea level in the SouthEast of Spain at the Observatorio de Sierra Nevada in Granada. The telescope was equipped with the simultaneous four-channel photometer (uvby Strömgren 
photoelectric photometer). For more details see Breger et al. (2004, 2005). In 2002129 hours of data during 29 nights, and in 200324.9 hours during 8 nights were collected. This data set was selected for the final analyses because of its satisfactory small scatter. In total, this sums up to about 154 hours of observation time for both years.

In this paper it is implicitly assumed that the intrinsic values $m_{0}$ and $c_{0}$ of FG Vir are the same as the observed $m_{1}$ and $c_{1}$ ones. It is valid in the case of FG Vir because the reddening $\mathrm{E}(b-y)=0$ (see e.g. the reddening assuming the mean indices published in the catalogue of Rodríguez et al. (2000) for $\delta$ Scuti-type stars).

Moreover, the given data represent measurements in the uvby filters. I determined the $\beta$ values directly from the corresponding $(b-y)$ ones assuming the $\beta(b-y)$ relation from Crawford (1979) for standard A-type stars. This is valid when, as in the case of FG Vir (more or less),

a) the reddening is null and

b) the star is on the Main Sequence.

If a star is not exactly on the Main Sequence, then a correction for "luminosity" has to be taken into acccount. See the relation in Crawford (1979) when $\delta m_{1}$ is positive:

$$
(b-y)_{0}=2.946-\beta-0.1 \delta c_{1}
$$

In the case of FG Vir $\delta c_{1}=0.09$; hence a difference of about -0.009 mag occurs to the corresponding $\beta$ values in the ZAMS which can be neglected with respect to the aims of my investigations, dealing with the testing of the trend of $m_{1}$ and $M_{V}$. Throughout this paper Crawford's (1979) transformation for the standard stars will be used for determining the corresponding $\beta$ values for each data record, which will be used for all the following analyses.

\section{Testing the temperature dependence of $m_{1}$ and $\delta m_{1}$}

Crawford (1979) published calibrations of the Strömgren parameters for Atype stars based on measurements of a large number of different stars. The pulsating star FG Vir changes its luminosity, surface temperature and log $g$ during a pulsation cycle. The mass, however, remains constant and the chemical abundance does not change either. Those are the prerequisites of this paper for checking the applicability of Crawford's (1979) calibration procedure through the change of the Strömgren parameter $m_{1}$ of the pulsating star FG Vir. In a first step the colour index $m_{1}(\beta)$ of FG Vir is compared to the colour index $m_{1}(\beta)$ of the standard stars (Hyades) as published by Crawford (1979). 
The method of testing the agreement of the colour indices is a linear fit of $m_{1}(\beta)$ from which the slope $k_{m}$ can be determined by:

$$
m_{1}(\beta)=\mathrm{a}_{\mathrm{m}}+\mathrm{k}_{\mathrm{m}} \beta .
$$

For better comparability of the two trends and taking into consideration the range of $\beta$ for FG Vir, the $m_{1}(\beta)$ values of the standard stars have also been linearized.

Fig. 1 shows the good agreement between the trends of $m_{1}$ for the Hyades and FG Vir caused by the pulsations. Let us denote for each $\beta m_{1 \mathrm{~m}}$ as the "mea-

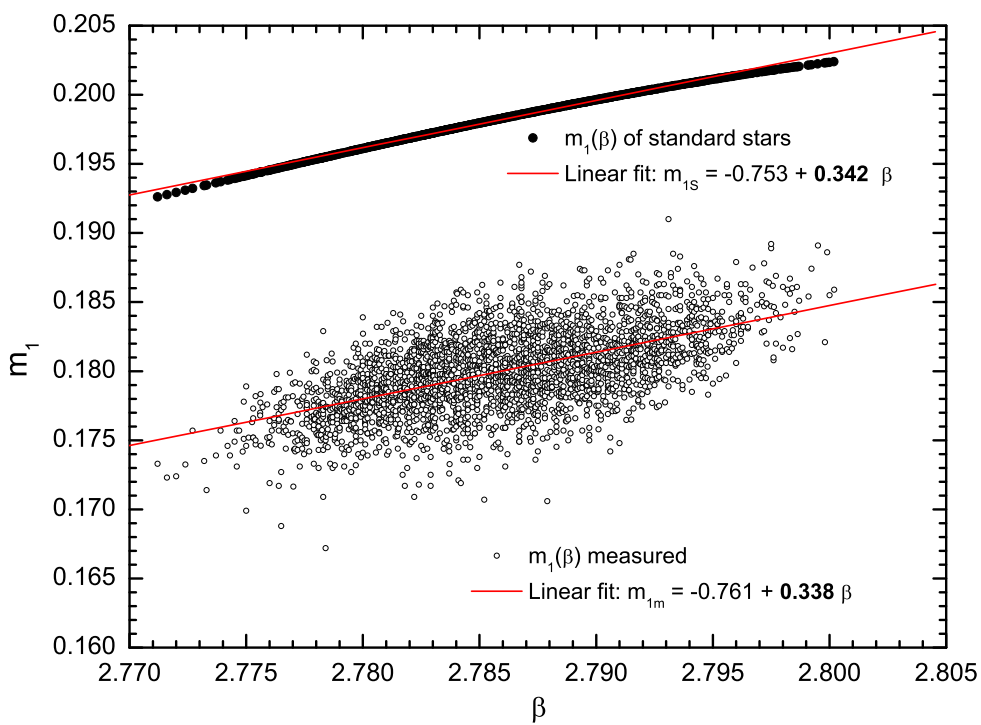

Figure 1: Comparison of the trend of $m_{1}$ of the standard stars with the trend of $m_{1}$ seen during pulsation of FG Vir. The shift between the two trend lines is caused by a different abundance between the Hyades and FG Vir. This has no effect to the calibration.

sured" values of FG Vir and $m_{1 \mathrm{~S}}$ as the values for the corresponding standard star. Crawford (1975) defined the blanketing parameter $\delta m_{1}(\beta)=m_{1 S}(\beta)$ $m_{1 \mathrm{~m}}(\beta)$. This parameter can be used for a consistency test. The constance of $\delta m_{1}$ over the pulsation cycle (Fig. 2) supports the conformity of the trend of $m_{1 \mathrm{~m}}(\beta)$ and the trend of $m_{1 \mathrm{~S}}(\beta)$. The conformity of both trends is also shown in Table 1. Data have been divided into 5 contiguous groups each comprising about 610 records. 


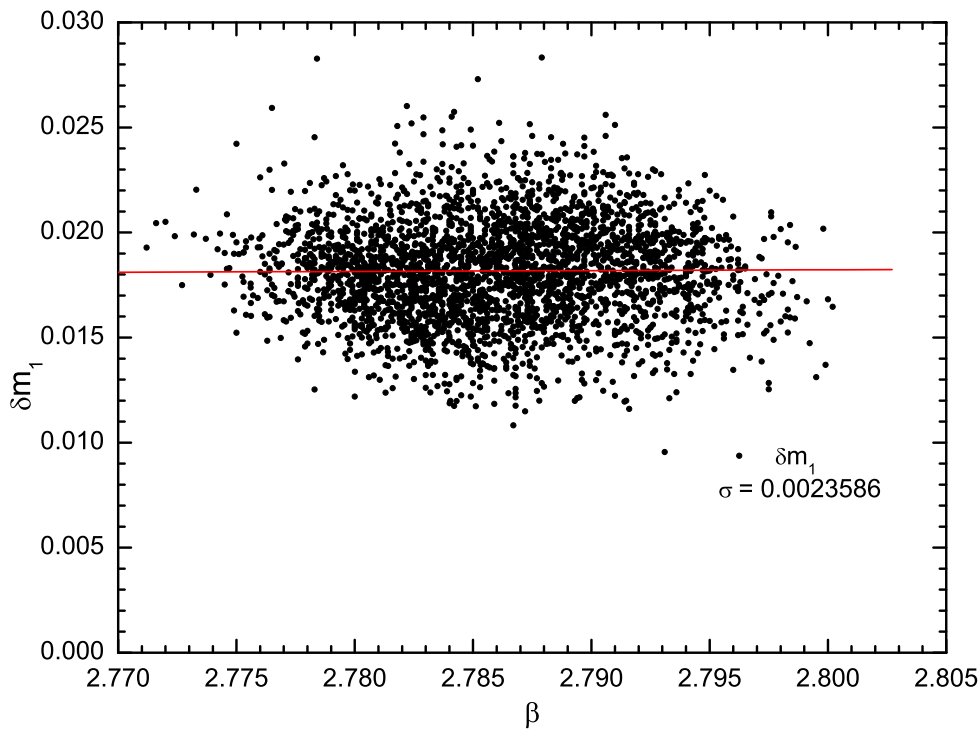

Figure 2: Consistency test based on $\delta m_{1}$

Table 1: Consistency test based on mean values of $\delta m_{1}$

\begin{tabular}{rrrr}
\hline \hline$[\beta]$ & {$[b-y]$} & {$\left[\delta m_{1}\right]$} & $\sigma$ \\
\hline 2.779 & 0.167 & 0.0182 & 0.0020 \\
2.783 & 0.163 & 0.0179 & 0.0025 \\
2.786 & 0.160 & 0.0180 & 0.0025 \\
2.789 & 0.157 & 0.0186 & 0.0025 \\
2.793 & 0.153 & 0.0181 & 0.0022 \\
\hline
\end{tabular}

If the size of the measured data sample is sufficiently large, a statement can be made with respect to the behaviour of the photometric index $m_{1}$ for $\delta$ Scuti stars pulsating with low amplitudes.

Evidently, the conformity of the trend of measured $m_{1}$ and the trend of calibrated $m_{1}$ depends on the abundances of the metals of the analysed star. Rodríguez et al. (1991) showed that differences in the behaviour of the $m_{1}$ variations in some stars with similar surface temperatures and gravities could be caused by different metallicities. Furthermore, Rodríguez (1991) pointed out that some authors assume a varying microturbulence velocity during a pulsation 
cycle responsible for the changing behaviour of $m_{1}$. This affects the strength of the metal-lines. Stankov (2002) showed that for the metal-poor star SX Phe no conformity of the trend of $m_{1}$ with the trend of the standard stars can be seen. Rodríguez (1991) explained this phenomenon with the existence of a different trend of $m_{1}$ for stars with low metallicities, referring to SX Phe stars explicitly, as a function of the $\delta m_{1}$-index variation and consequently also of the $m_{1}$-index variation. Over the pulsation cycle the variation of $m_{1}$ increases with decreasing metallicity. Önehag et al. (2009) investigated the calibration of Strömgren uvby $\mathrm{H} \beta$ photometry for $\mathrm{G}$ and $\mathrm{F}$ stars and tested the influence of temperature, gravity and metallicity on the $m_{1}$ index. The authors reported similar results as those found in this work on A-type stars.

\section{Testing the temperature dependence of $c_{1}(\beta)$}

The Balmer discontinuity-index $c_{1}$ provides an estimate for the luminosity of a star from the computed difference $\delta c_{1}$ relatively to stars at the ZAMS. For High Amplitude $\delta$ Scuti stars studies of the variations of the colour indices $c_{1}$ and $\delta c_{1}$ have been performed by Rodríguez et al. (1993 and 2003) and for SX Phe by Rolland et al. (1991). Also a study of the trend of $\delta c_{1}$ can be found in Stankov et al. (2002) for SX Phe. In their paper a value of $\delta \mathrm{V} / \delta c_{1}=9.8$ for SX Phe is derived which is in good agreement with the value given in Crawford's (1975) paper for F-type stars.

For FG Vir the procedure, as specified by Stankov (2002), provides a value for $\delta y / \delta c_{1}=10.3$ as can be seen in Fig. 3. Note that by definition $y=V$. In this plot the band of $y$ is segmented into 9 groups of equidistant width.

The above discussions relate to the behaviour of the Strömgren-index $c_{1}$ for the single star FG Vir. The paper of Crawford (1979) describes the calibration of $c_{1}$ at constant effective temperature $T_{\text {eff }}$ and his results are based on the analysis of different stars with different masses. The dependence of the luminosity on the stellar mass will be shown in the following sections about the $M_{V}$ calibration. The differences in mass have an influence (even if it is low) on the value of $\log g$ and consequently also on $c_{1}$.

\section{Relation between $\delta c_{1}$ and $\delta m_{1}$ in A-type stars}

Crawford (1979) examined the correlation between the trends of $\delta c_{1}$ and $\delta m_{1}$ for A-type stars and found a small relationship between these two indices: "... any star with a large $\delta c_{1}$ has a larger-than-average $\delta m_{1}$. Also, those stars with" more "negative $\delta m_{1}$ (the Am-type stars) have smaller-than-avergage $\delta c_{1}$. The 


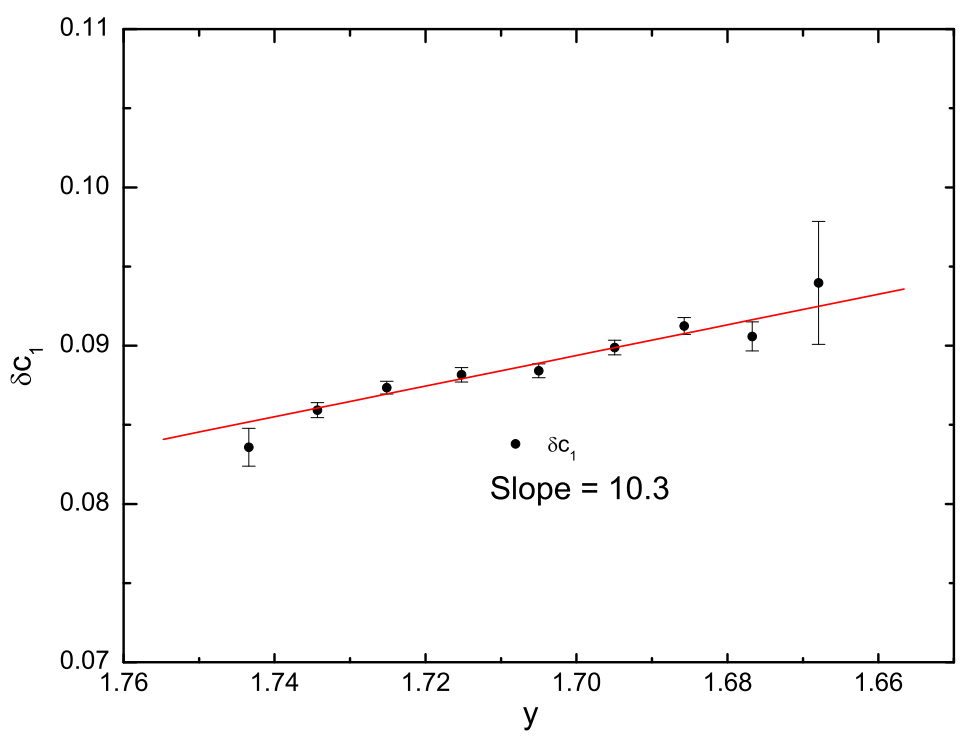

Figure 3: $\delta c_{1}$ versus $y$ divided into 9 groups. This shows the agreement with the expected value of a slope of 9.0 for A-type stars derived for different stars by Crawford. The agreement can be improved by taking the mass effect into account.

values of both indices for FG Vir are located in a very " normal range. In Fig. 5 of Crawford's paper a trend can be found. Fig. 4 shows the relation of $\delta c_{1}$ and $\delta m_{1}$ for FG Vir. A linear fit to the FG Vir data in Fig. 4 lies completely within the cloud of points shown by Crawford (1979) in Fig. 5 of his paper. There is a clear trend in the sense of larger $\delta c_{1}$ values as the $\delta m_{1}$ values are also larger. This is the same trend as in the Crawford paper, but it is evident that the interpretation must be quite different (if existing) for a pulsating star. What does this trend mean in the case of FG Vir? If we inspect Fig. 2, $\delta m_{1}=$ $\delta m_{1}(\beta)$, we can either assume whether the "central" cloud of points (centred at $\beta=2.785 \mathrm{mag}$ ) is "only" due to "dispersal" of the data points or whether it is related to the pulsation phase of the star. And there are some more questions addressing the relation between the investigated properties and the phase. Questions with this respect are not subject of the current paper. FG Vir is a multiperiodic star and thus makes investigations very complex. 


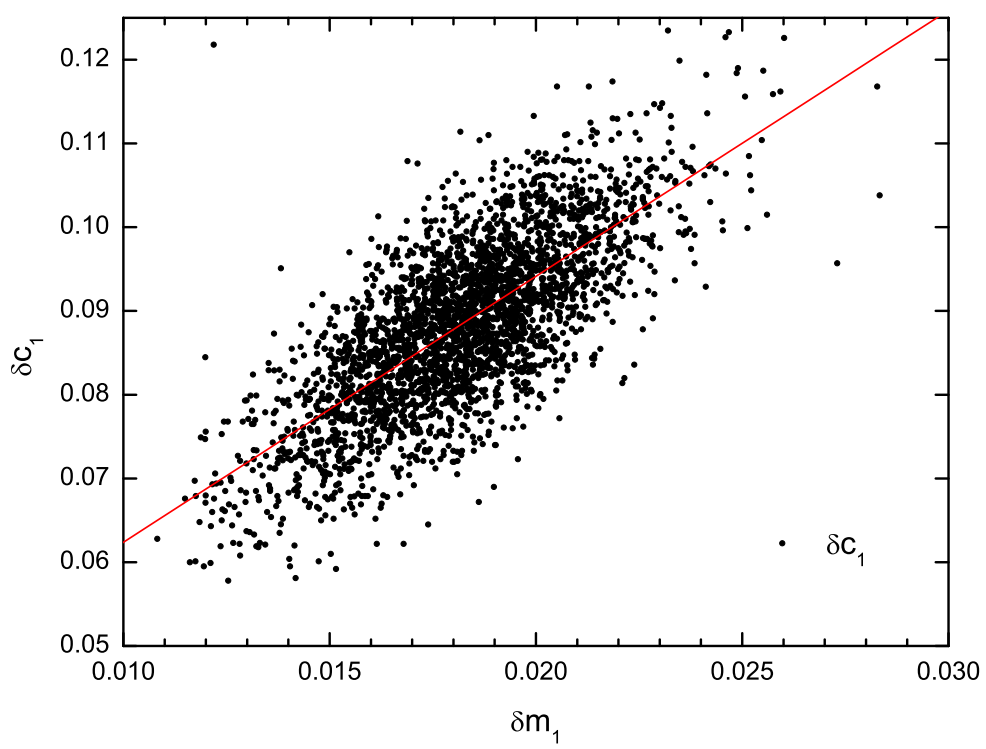

Figure 4: Relation between $\delta \mathrm{c}_{1}$ and $\delta \mathrm{m}_{1}$ for the measured values

\section{Testing the temperature dependence of $M_{V}(\beta)$}

In this section the trend between the colour measurements $y(\beta)$ of FG Vir and the calculated values for the absolute magnitude $M_{V}(\beta)$ according to Crawford's (1979) calibration are compared. This calibration determines $M_{V}(\beta)$ by means of $M_{V}($ ZAMS, $\beta)$ denoting the stars at the position of the ZAMS and $\delta c_{1}(\beta)$ according to:

$$
M_{V}(\beta)=M_{V}(\text { ZAMS, } \beta)-f . \delta c_{1}(\beta) .
$$

$M_{V}($ ZAMS, $\beta)$ and $c_{1}$ (ZAMS, $\beta$ ) are taken from Table I in Crawford's (1979) paper. Crawford proposes $f=9$ for A-type stars and

$$
\delta c_{1}(\beta)=c_{1}(\beta)-c_{1}(\text { ZAMS, } \beta) .
$$

Hypothesis: the trend of $M_{V}(\beta)$, determined by Crawford's (1979) calibration as outlined above, confirms the trend of the measurements of $y(\beta)$ for FG Vir, i.e., $M_{V}$ fits the measurements of $y=V$. 
To verify this hypothesis the calculated values of $M_{V}$ are fitted linearly to obtain the trend for $M_{V}(\beta)$, which is then compared to the trend of the measurements of $y(\beta)$ for FG Vir (also based on a linear fit).

Result: the initially postulated hypothesis for the trend of $M_{V}(\beta)$ cannot be confirmed under the strict application of Crawford's $(1979,1987)$ calibration procedure and Crawford's data for the position of the ZAMS. The fit between $M_{V}(\beta)$ and $y(\beta)$ of FG Vir is unsatisfactory.

The poor agreement between both trends can be seen immediately in Fig. 5 .

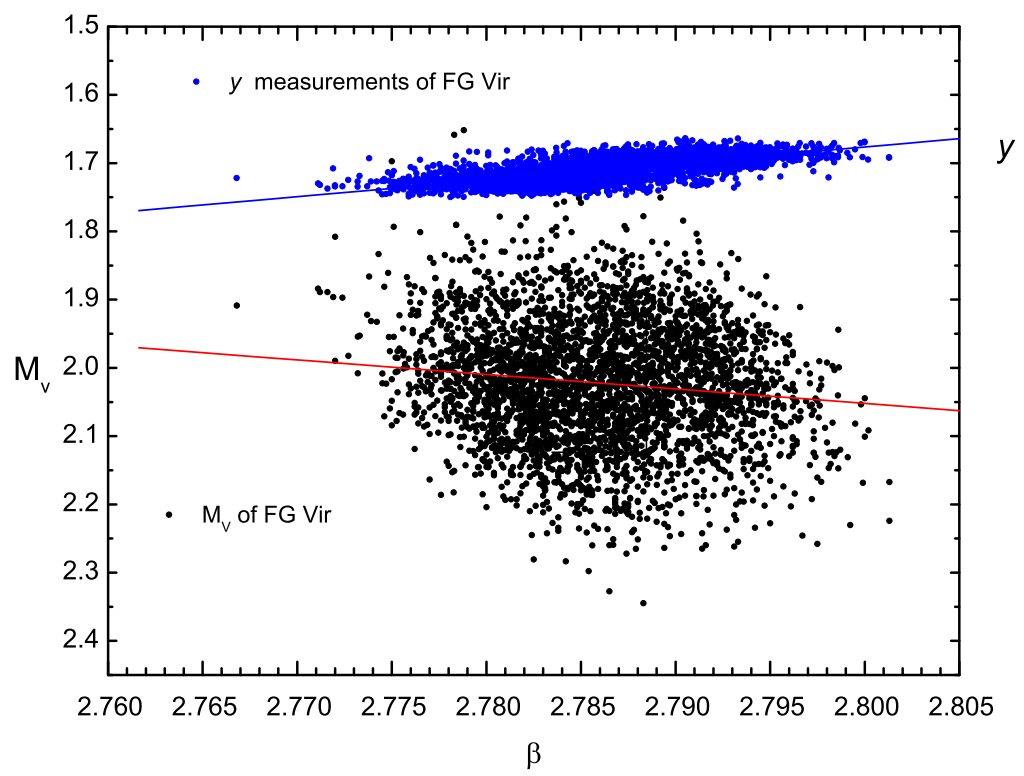

Figure 5: Comparison of the trend line of $M_{V}(\beta)$ according to Crawford's calibration and the measured values of $\mathrm{y}(\beta)$.

I examined which changes to the calibration procedure are necessary to improve the agreement between the trends of $M_{V}(\beta)$ and $y(\beta)$. The first step was to modify the factor $f$. Although a reduction of this factor improves the agreement between both fits, an optimal value for $f$ could not be found.

The next question was whether a change to the adopted position of the ZAMS provides a better fit and how we can determine the "real" position of the ZAMS.

Mermilliod (1981) published a table for $M_{V}(Z A M S, B-V)$ for the posi- 
tion of the ZAMS in the Johnson UBV system and added the remark: "...The present material may be used to redetermine the ZAMS." The table shows a different temperature dependency for $M_{V}(Z A M S)$ compared to the one published by Crawford (1979). A transformation as indicated by Caldwell (1993) allows the representation of the table $M_{V}($ ZAMS, $B-V)$ in a form suitable for the Strömgren uvby $\beta$ photometric system. The substitution of Crawford's position of the ZAMS by Mermilliod's position of the ZAMS proved to be very effective for improving the agreement of the trends between $M_{V}(\beta)$ and $y(\beta)$. For the calibration of the Strömgren parameters Crawford (1979) selected the open clusters $\alpha$ Per, the Pleiades, IC 4665, the Hyades and data from the Coma cluster, UMa and Praesepe. In order to determine the position of the ZAMS, Mermilliod (1981) selected the open clusters $\alpha$ Per, the Pleiades, IC 4665 and additional open clusters younger than the Hyades.

With this modification to the position of the ZAMS the agreement between both fits improves substantially, but is still not perfect.

Fig. 6 illustrates the disagreement between both positions of the ZAMS definitions in the temperature region of FG Vir.

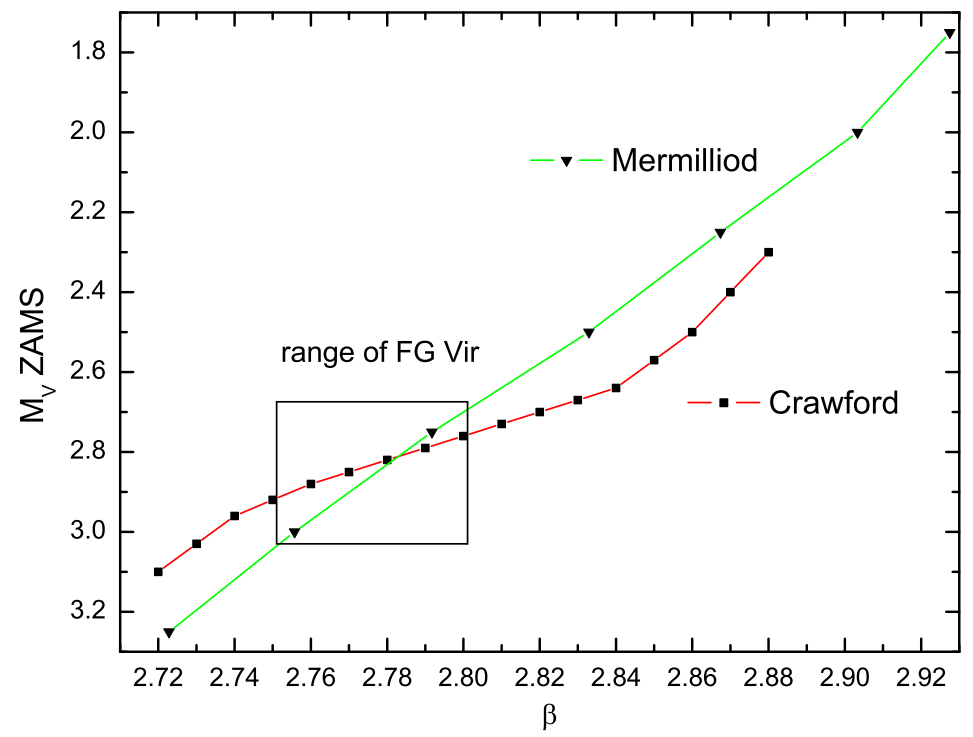

Figure 6: Comparison between the position of the ZAMS of Crawford and the position of the ZAMS of Mermilliod. The rectangle marks the temperature region of FG Vir. 
We find that Mermilliod's values for the $M_{V}$ values of the position of the ZAMS, $M_{V}($ Mer, $\beta)$, and a reduction of the factor $f$ in the calibration formula provides a better fit for $M_{V}$ of FG Vir. This allows us to draw the following conclusion:

The optimal calibration leading to the best agreement between the trend of the measured values $y(\beta)$ and the trend of the calibrated $M_{V}(\beta)$ for FG Vir is obtained by the following equation (see Fig. 7):

$$
M_{V}=M_{V}(\operatorname{Mer}, \beta)-7.1 \delta c_{1}
$$

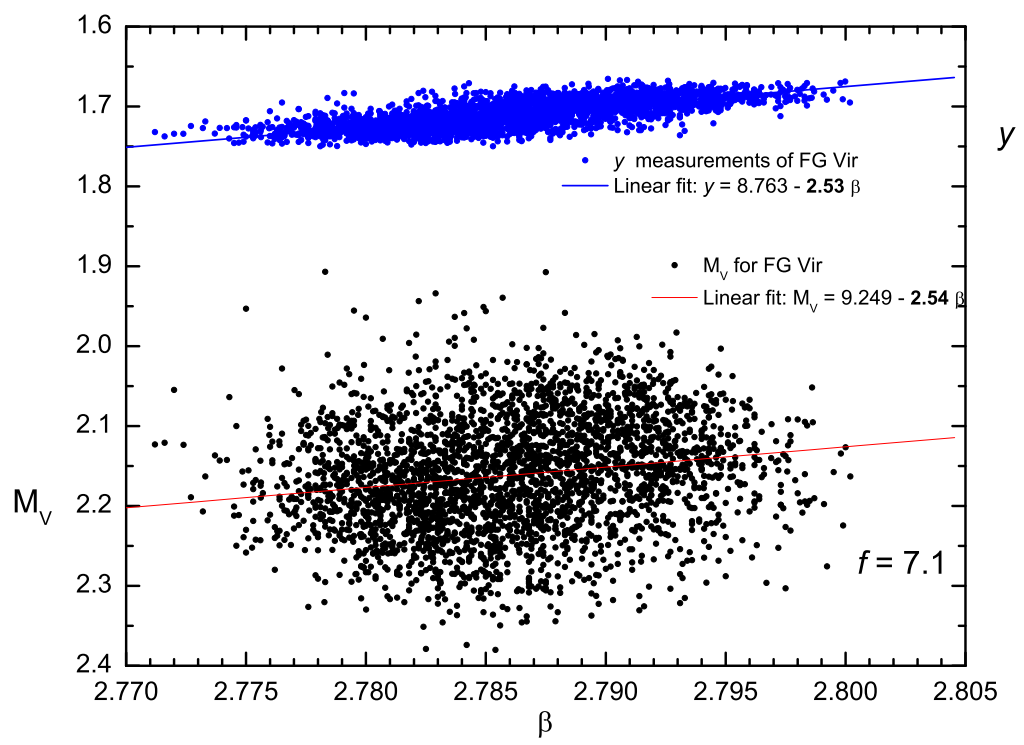

Figure 7: Comparison of the trend line for the measured values of $y(\beta)$ and trend line of $M_{V}(\beta)$ calibrated with $M_{V}($ ZAMS, $\beta)$ as indicated by Mermilliod and the factor $f$ reduced to $f=7.1$.

These results, however, are still tentative since the effect of the mass has still to be taken into account (see section 7.). Hence, the next step will be to consider the effect of different masses. Are the different values for the factor $f$ in Crawford's (1979) calibration and in the optimal calibration obtained in this paper a result of mass differences? 


\section{Effect of mass on the fundamental parameters of a main se- quence star}

In this paragraph the effect of the mass difference between FG Vir and main sequence stars with the same luminosity and temperature on $\log g$ and $c_{1}$ will be investigated.

Stars located on the main sequence are in similar stages of their evolution and therefore exhibit a similar internal structure. Therefore, a mass, $\mathcal{M}$, is in accordance with a specific absolute bolometric magnitude $M_{\text {bol }}$ and hence a corresponding luminosity L. In Crawford's (1979) calibration procedure this relationship was kept in mind for A-type stars implicitly for the absolute magnitude $M_{V}$ (and with it the determination of $L$ and $M_{\text {bol }}$ ). The analysis of my paper is focused on the application of the calibration relation of Strömgren photometry to determine the luminosity $L$ of the single star FG Vir, which does not change its mass $\mathcal{M}$. During the pulsation cycle of the star mainly the variations of the fundamental parameters $T_{\text {eff }}$ (included in $b-y$ and $\beta$ ), $\log g$ (included in $c_{1}$ ), $L$ (included in $y$ or in $M_{V}$, determined according to the calibration of Crawford) as well as the radius $R$ are analysed. The mass $\mathcal{M}$ of FG Vir will remain unchanged during the pulsational variations and hence causes a different constellation in comparison with a normal main sequence star (this can also be seen as a perturbation of the equilibrium of the star). One can also make a prediction which mass $\mathcal{M}$ is consistent with the respective luminosity $L$ for every pulsation phase and hence find out the difference of the mass of FG Vir compared to a "nominal value" (or position of an equilibrium model star). In other words, which should be the mass of the star FG Vir in every phase of the pulsation cycle in comparison with an unperturbed star?

I made quantitative estimates of the mass differences and their effect especially on the values of $\log g$ based on the same models and software as used by Lenz et al. (2008). Metallicity and rotational velocity of the stars are selected in accordance with FG Vir. The evolutionary tracks in Fig. 8 for different masses allow an estimate of the difference between the mass of FG Vir and the "nominal mass" of a corresponding unperturbed main sequence star. During each pulsation phase $\log L$ of FG Vir has to be identical to $\log L$ of a corresponding main sequence star. Furthermore, by definition the surface temperature $T_{\text {eff }}$ is also the same for both stars. Taking into account the above constraints based on the relations between the fundamental parameters of a star, the following relation between surface gravity $g$ and mass $\mathcal{M}$ can be derived:

$$
g_{\mathrm{FG}} / g_{\mathrm{MS}}=\mathcal{M}_{\mathrm{FG}} / \mathcal{M}_{\mathrm{MS}},
$$

where the subscript FG denotes FG Vir and MS a corresponding main sequence star. The deviation $\left(g_{\mathrm{FG}}-g_{\mathrm{MS}}\right)$ caused by the mass difference between 
16 Analyses of Crawford's uvby $\beta$ calibrations using the pulsational variations of FG Vir

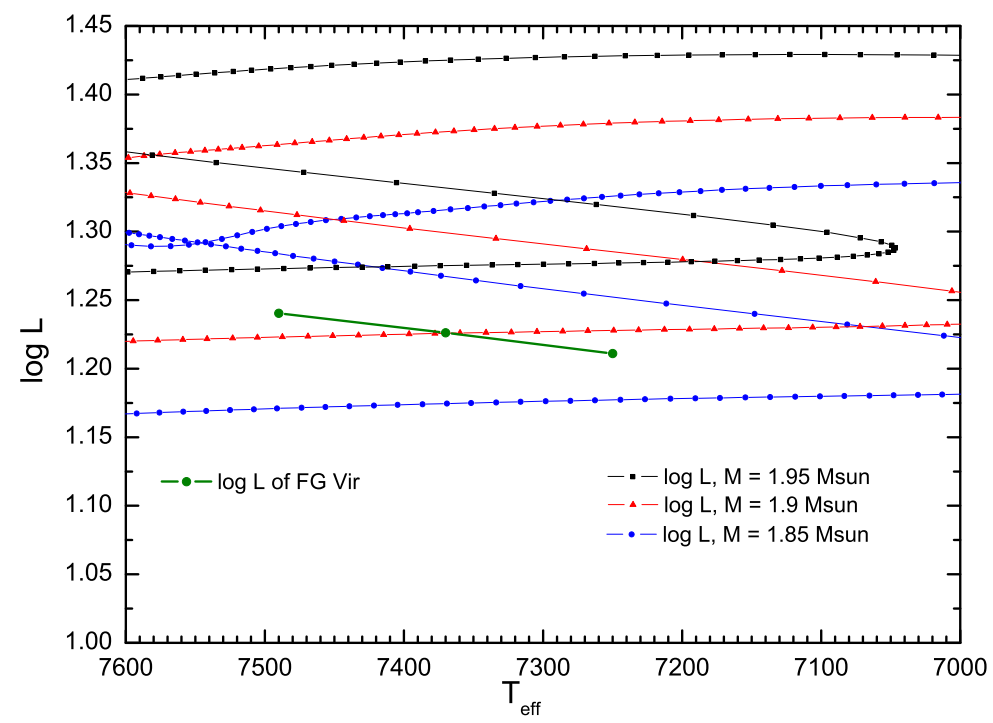

Figure 8: Comparison of evolutionary tracks for 3 main sequence stars with 3 different masses and a corresponding curve spanned by FG Vir with more than 75 frequencies $\left(\log L=\log L / L_{\odot}\right)$.

FG Vir and a main sequence star with identical luminosity and temperature has an equivalent $\delta c_{1}$ value of $\left(c_{1 \mathrm{FG}}-c_{1 \mathrm{MS}}\right)$. By means of the $c_{1}(\beta)$ diagram the corresponding $c_{1}$ values of the respective main sequence star can be determined. Metallicity and microturbulence of the stars in the diagram are selected in accordance with FG Vir. A description of the used atmosphere model grids can be found in Nendwich et al. (2004) and Heiter et al. (2002). Table 2 highlights 3 points of the temperature range (mean, maximum and minimum) caused by the pulsational variation range of FG Vir comparing the measured value of $c_{1 F G}$ to the respective "nominal value" $c_{1 M S}$ of the corresponding main sequence star. By definition the deviation of the mean value is chosen to be 0.0 .

In the range of the temperature variation of $\mathrm{FG}$ Vir the maximal deviation of the nominal value $c_{1 \mathrm{MS}}$ of the corresponding main sequence star is less than $5.5 \%$. One can substitute FG Vir's $c_{1 F G}$ by $c_{1 M S}$ of the corresponding main sequence star and compare the trends of the measured $y$ values and $M V$ calculated according to Crawford's procedure, for FG Vir with the ZAMS 
Table 2: Selected points of the deviation for $c_{1}$ between FG Vir and a main sequence star

\begin{tabular}{rrrrr}
\hline \hline$T_{\text {eff }}$ & $\beta$ & $c_{1 \mathrm{FG}}$ & $c_{1 \mathrm{MS}}$ & $c_{1 \mathrm{FG}}-c_{1 \mathrm{MS}}$ \\
\hline 7490 & 2.800 & 0.860232 & 0.861322 & 0.00109 \\
7370 & 2.786 & 0.840347 & 0.840347 & 0.00000 \\
7250 & 2.771 & 0.819042 & 0.817962 & -0.00108 \\
\hline
\end{tabular}

of Mermilliod in place of Crawford's ZAMS. This leads to a good agreement between both trends.

A linear function can be derived from Table 2 for the determination of $c_{1 \mathrm{MS}}$ as soon as the "measured" $c_{1 F G}$ value is known.

\section{Conclusions}

The current work presents an analysis of the behaviour of the two Strömgren indices $c_{1}$ and $m_{1}$ based on 3068 records of uvby Strömgren photometric data for the $\delta$ Scuti star FG Vir. Furthermore, the applicability of Crawford's (1979) calibration procedure for the determination of the trend of the absolute magnitude $M_{V}$ during pulsations is critically tested by comparing the $M_{V}$ calibration of FG Vir with the corresponding observed values.

The testing of the trend of $m_{1}$ of FG Vir confirms that the metallicity is not varying during the pulsation cycle. It fits with the trend of the standard stars. This can be seen by the fact that the blanketing parameter $\delta m_{1}$, which characterizes the metal content of the star, remains constant. Additionally to previous results for High Amplitude $\delta$ Scuti stars (Rodríguez et al. 1991), the confirmation is possible with the given data even for a $\delta$ Scuti star with low amplitude.

The testing of the trend of the absolute magnitude $M_{V}$, determined according to Crawford's (1979) calibration procedure, shows a poor agreement with the trend of the measured intrinsic colour $y$. The published calibration of $M_{V}$ does not fit the measured values of FG Vir. I outline an approach to reach a satisfactory agreement by taking into account the effect of different masses on the trends of $\log g$ and $c_{1}$, especially by substituting the position of the ZAMS as outlined by Crawford (1979) with the position of the ZAMS as defined by Mermilliod (1981). Mermilliod indicates that a redefinition of the position of the ZAMS will be necessary based on his observations. The current work fully supports this statement. 
Acknowledgments. The author thanks Michel Breger for suggesting the subject of this research, for guiding the progress of the research, and for giving invaluable assistance and suggestions during the initial and final analysis of the data in this investigation.

The uvby data were collected at SNO (Sierra Nevada Observatory).

This work was supported by the Austrian Fonds zur Förderung der wissenschaftlichen Forschung (FWF).

\section{References}

Breger, M., Rodler, F., Pretorius, M. L., et al. 2004, A\&A, 419, 695

Breger, M., Lenz, P., Antoci, V., et al. 2005, A\&A, 435, 955

Caldwell, J. A. R., Cousins, A. W. J., Ahlers, C. C., et al. 1993, SAAO, 15, 1

Crawford, D. L. 1966, IAUS, 24, 170

Crawford, D. L. 1975, AJ, 80, 955

Crawford, D. L. 1978, AJ, 83, 48

Crawford, D. L. 1979, AJ, 84, 1858

Heiter, U., Kupka, F., van 't Ver-Mennert, C., et al. 2002, A\&A, 392, 619

Lenz, P., Pamyatnykh, A. A., Breger, M., \& Antoci, V. 2008, A\&A, 478, 855

Lester, J. B., Gray, R. O., \& Kurucz, R. L. 1986, ApJS, 61, 509

Mermilliod, J. C. 1981, A\&A, 97, 235

Moon, T. T., \& Dworetsky, M. M. 1985, MNRAS, 217, 305

Nendwich, J., Heiter, U., Kupka, F., et al. 2004, CoAst, 144, 43

Nissen, P. E., Twarog, B. A., \& Crawford, D. L. 1987, AJ, 93, 634

Önehag, A., Gustafsson, B., Eriksson, K., \& Edvardsson, B. 2009, A\&A, 498, 527

Perry, C. L., Olsen, E. H., \& Crawford, D. L. 1987, PASP, 99, 1184

Philip A.G.D., \& Egret, D. 1980, A\&AS 40, 199

Rodríguez, E., Rolland, A., López de Coca, P., \& Garrido, R. 1991, A\&A, 247, 77

Rodríguez, E., Rolland, A., López de Coca, P., et al. 1992, A\&AS, 96, 429

Rodríguez, E., Rolland, A., \& López de Coca, P. 1993, A\&AS, 101, 421

Rodríguez, E., Rolland, A., Garrido, R., et al. 1998, A\&A, 331, 171

Rodríguez, E., López-González, M. J., P., \& López de Coca, P. 2000, A\&AS, 144, 469

Rodríguez, E., Arellano Ferro, A., Costa, V., et al. 2003, A\&A, 407, 1059

Rolland, A., Rodríguez, E., López de Coca, P., et. al. 1991, A\&AS, 91, 347

Smalley, B., \& Dworetsky, M. M. 1993, A\&A, 271, 515 
Smalley, B. 1993, A\&A, 273, 391

Stankov, A., Sinachopoulos, D. A., Elst, E., \& Breger, M. 2002, CoAst, 141, 72

Strömgren, B. 1963, QJRAS, 4, 8

Strömgren, B. 1966, ARA\&A, 4, 433 
